\title{
Role of Insulin/Glucagon Ratio and Cell Redox State in the Hyperglycaemia Induced by Exposure to Electromagnetic Fields in Rats.
}

Gabriel Martiñón-Gutiérrez

National Autonomous University of Mexico

María Luna-Castro

National Autonomous University of Mexico

Rolando Hernández-Muñoz ( $\nabla$ rhernand@ifc.unam.mx )

National Autonomous University of Mexico

\section{Research Article}

Keywords: Glucose metabolism, Insulin, Glucagon, Oxidized redox state, serum lipids

Posted Date: February 5th, 2021

DOl: https://doi.org/10.21203/rs.3.rs-148773/v1

License: (c) (i) This work is licensed under a Creative Commons Attribution 4.0 International License.

Read Full License 


\section{Abstract}

The exposure to extremely low-frequency electromagnetic fields (EMFs) with a might adversely affect the endocrine system and reproductive functions. Nonetheless, the use of EMFs in the form of magnetotherapy exerts beneficial actions in human medicine, but also affecting glucose and lipids metabolism, inducing hyperglycaemia and a "diabetic" response. Therefore, we used fed or fasted male rats for a single exposure to $60-\mathrm{Hz}$ EMFs, determining serum levels of glucose, lipids, and indicators of cellular redox state and energy parameters. A single exposure to EMFs induced hyperglycaemia in both, fed and fasted rats, accompanied by an attenuated second serum insulin peak. In addition, EMFs also decreased serum levels of free fatty acids and lactate, while they increased those of pyruvate and acetoacetate. Changes in blood glucose level were related with a more oxidized cellular redox state and with the insulin/glucagon ratio. Moreover, rat's glucose metabolism also coincided with variations in insulin and glucagon secretion, probably depending on cell redox state. The EMFs effects were not modified in animals previously subjected to a chronic exposure (14 days). In conclusion, increased serum glucose levels and glucose metabolism induced by EMFs exposure were closely related with the cellular redox state and with the insulin/glucagon ratio.

\section{Introduction}

Whenever electrical current flows, both electrical and magnetic fields are generated, which are known as electromagnetic fields (EMFs) [1]. These extremely low-frequency EMFs have a long wavelength and occupy the range between 3 and $300 \mathrm{~Hz}$ [2], its exposure might adversely affect the endocrine system and reproductive functions, as well as the fetal development of animals [3].

Despite this scenario, some studies highlight the positive effects of magnetic field therapy, in particular, in the rehabilitation of post-stroke patients and cancer treatment, mainly in combination with an anticancer drug $[4,5]$. From here, the use of EMFs, in particular of that named "magneto-therapy", has had a notable increase in the last times, principally in rehabilitation treatment, where it provides a non-invasive and safe method to locally treat the site of injury, ameliorating the pain, or in other systemic diseases as diabetes type 2, where inflammation occurs as a pathogenic feature [6, 7]. Moreover, EMFs are seen as a prospective, noninvasive, and safe physical therapy strategy to accelerate bone repair, by stimulating signaling cascades, which effectively promote osteogenesis and angiogenesis in an orchestrated manner [8].

In this regard, EMFs have been found to reduce the release of pro-inflammatory cytokines such as tumour necrosis factor- $a$ (TNF-a), interleukin (IL)-1 $\beta$, and IL- 6 in LPS-activated N9 microglial cells, suggesting that EMFs could represent a potential therapeutic approach in cerebral ischemic conditions [9]. In fact, pulsed EMFs exposure becomes a potential therapeutic option, based on its angiogenesis-promoting properties [10], and its ability to reduce infarct size and neuro-inflammation [11], through decreasing pro-apoptotic mediators and increased pro-survival molecules [12], in response to activated signalling pathways induced by EMFs exposure [13]. 
The exposure of cells to a $50-\mathrm{Hz}$ magnetic field could induce EGF receptor clustering and phosphorylation; EMFs can accelerate neural differentiation of bone marrow-derived macrophages via EGF receptor activation [14]. Therefore, changes in EGFR status are also accompanied by changes in the level of reactive oxygen species (ROS) after EMFs exposure $[15,16]$, increasing enzymatic antioxidant activity and improve functional and mental [17].

On the other hand, exposure to EMFs could participate in the response to stress by affecting cell metabolism especially that linked to metabolic energy production. EMFs suppress lactate anabolism, which could depend on the adrenergic status of the animal. Application of EMFs to hind limb ischemic rats did not significantly increase blood levels of lactate and glucose, but decreased those levels of free fatty acids (FFA) [18]. However, it has been reported also that, in normal animals subjected to stress, 50 $\mathrm{Hz}$ EMFs exposure tends to decrease plasma ACTH and glucose, and significantly decreases plasma lactate levels in the stressed rats [19]. In addition, these EMFs decrease plasma levels of total cholesterol and phospholipids, and downregulate liver diacylglycerol acyltransferase-2 mRNA expression [20]. The mechanisms for the effects of EMFs on glucose and lipid metabolism are not well understood yet, but effects on insulin secretion could also be involved.

Long time ago, it was reported that, in rats chronically exposed to uniform constant EMFs, blood glucose slightly increased, the release of insulin decreased, and the glucagon content increased showing a temporarily diabetic-like response in the treated-rats [21]. These effects were also confirmed with the exposure to EMFs at $5 \mathrm{mT}$ to HITT15 cells, where glucose-stimulated insulin secretion, ATP/ADP ratio, membrane depolarization, and cytosolic free calcium ion concentration, were decreased [22]. Therefore, the serum levels of glucose and the glucose-stimulation of insulin secretion could depend on the cellular metabolic conditions and the protocol of EMFs treatment.

The present work is an attempt to gain some insight in the metabolic responses of fed or fasted male rats to a single exposure to extreme-low frequency EMFs, measuring serum levels of glucose, lipids, and indicators of cellular redox state and energy parameters, as well as performing a metabolic tracing of glucose in the whole animal. Our data indicate that EMFs induced a hyperglycaemic state in both, fed and fasted rats, accompanied by a drastic attenuation of a second serum insulin peak. Indeed, increased serum glucose levels were closely related with the cellular redox state and with the insulin/glucagon ratio.

\section{Results}

\section{Effects of the single exposure to EMFs in glucose, lipids, and redox pair-metabolites in fed and fasted rats.}

Animals were subjected to a 15 min-exposure of $60 \mathrm{~Hz}$-EMFs (time zero), and euthanized immediately after obtaining blood samples. In fed animals, the EMFs did not affect significantly serum level of glucose or that of TAG, but decreased largely FFA serum levels (Fig. 1A). Moreover, EMFs exerted an 
opposite effect in the redox-pair metabolites: lactate was decreased and pyruvate was drastically increased in the serum; in addition, serum levels of AcAc were also significantly decreased (Fig. 1A).

In overnight fasted animals, in which the metabolic environment was changed, decreasing serum level of glucose, increasing those of FFA, and lactate and ketone bodies, the exposure to EMFs had not significant effects on these parameters, except that EMFs also decreased levels of FFA (Fig. 1B). Then, depending on the metabolic scenario, a single exposure to EMFs exerted significant metabolic changes in rats.

\section{Time-course of EMFs effects on glucose, lactate, and pyruvate in fed and fasted rats.}

Since we did not find higher levels of glucose in animals subjected to a 15 min-exposure of $60 \mathrm{~Hz}-\mathrm{EMFs}$, as previously reported [21], we looked for a time-course of EMFs effects on some parameters. In fed animals, serum levels of glucose increased at $15 \mathrm{~min}$ after exposure termination, returned to the control levels (30 min), and started again to increase $60 \mathrm{~min}$ after exposure (Fig. 2A). In these animals, lactate was early decreased, and this significant diminution was maintained, thereafter ( $3 \mathrm{~h}, \mathrm{Fig}$. 2B). On the contrary, EMFs promoted three peaks of augmented serum levels of pyruvate at 0,30 , and $180 \mathrm{~min}$ (Fig. 2C), where the lactate/pyruvate ratio was largely decreased (not shown).

Interestingly, in animals with an overnight fast, a hyperglycaemic status was observed after exposure to $60 \mathrm{~Hz}$-EMFs, reaching the same higher blood levels for glucose as in fed animals, at the end of the experiment (Fig. 2A). However, lactate levels (which were much higher in these animals) did not decrease, but rather showed two increasing peaks at 30 and $180 \mathrm{~min}$ after treatment (Fig. 2B). Also interesting, EMFs promoted two increments of serum pyruvate at 30 and $120 \mathrm{~min}$ (Fig. 2C). Therefore, increases of serum glucose seem to coincide with lower lactate/pyruvate ratios (more oxidized state) after EMFs treatment (Fig. 2).

\section{Curve of tolerance to glucose and levels of insulin and glucagon in rats treated with EMFs.}

Another set of rats were fasted overnight, administered with a glucose load of $2 \mathrm{~g} \cdot \mathrm{kg}^{-1}$, and subjected to a 15 min-exposure of $60 \mathrm{~Hz}$-EMFs. Control (sham) animals depicted a "typical" absorption and further decay of blood levels of glucose assumed to be due to its utilization. In rats treated with EMFs, we noted that, during the first 90 min, glucose tolerance was similar to that of the control group, but, at 120 min after glucose administration, a robust hyperglycaemia occurred, which could be considered as a "diabetic-like response" curve (Fig. 3A). In this context, control animals elicited two peaks of insulin secretion, the first at $15 \mathrm{~min}$ and the second $2 \mathrm{~h}$ after treatment. Although EMFs indeed increased the first peak of insulin when compared with control animals, the second insulin peak was practically abolished by the treatment with EMFs (Fig. 3B). Interestingly, in control and treated animals, serum levels of 
glucagon showed an opposite pattern of release when compared to that of insulin. However, during the first $30 \mathrm{~min}$ of the tolerance curve, animals treated with EMFs had the lowest serum levels of this hormone (Fig. 3C). Therefore, the insulin/glucagon ratio could be involved in the hyperglycemic effect of EMFs in these rats.

\section{In vivo oxidation of U-(14C)-glucose and its incorporation into glycogen and glycerol-containing lipids in rats treated with EMFs.}

Another set of fed rats received $0.5 \mathrm{mg}$ of glucose-containing $4 \mu \mathrm{Ci}(148 \mathrm{mBq})$ of $\left(\mathrm{U}-{ }^{14} \mathrm{C}\right)$-glucose, and was subjected to a 15 min-exposure of $60 \mathrm{~Hz}$-EMFs. Animals exposed to EMFs showed an increased glucose oxidation, as reflected by the production of ${ }^{14} \mathrm{CO}_{2}$ (Fig. 4A), which was accompanied by a tendency of decreasing blood level of ${ }^{14} \mathrm{C}$-glucose (Fig. 4B) 30 min after EMFs treatment. Interestingly, synthesis of liver glycogen, as assessed by incorporation of glucose into glycogen, was decreased at 120 min after exposure (Fig. 4C), coinciding with the abatement of the second peak of insulin secretion (Fig. 3B). As to muscular glycogen, treatment with EMFs induced two decrements in its synthesis, at 30 and 120 min, respectively (Fig. 4D); these effects also seemed to be related with the initial serum insulin (Fig. 3B), as well as with the augmented glucose oxidation (Fig. 4A). Moreover, whereas EMFs promoted lipogenesis (as assessed by FFA esterification to ${ }^{14} \mathrm{C}$-glycerol) in the liver (120 min; Fig. 4E), in the epididymal adipose tissue there was a transient decrease of the rate of lipogenesis 60 min after EMFs exposure (Fig. 4F).

\section{Correlations among serum glucose levels, insulin, glucagon, and redox-pair metabolites.}

We looked for possible relationships between the hyperglycaemic status induced by EMFs exposure and the serum levels pancreatic hormones, as well as with redox-pair metabolites (Fig. 5). The levels of glucose in control and treated rats correlated straight with fluctuations in insulin levels $(r=0.687, p<0.01$; Fig. $5 A$ ), with those of glucagon ( $r=0.403, p<0.05$; Fig. $5 B)$, and much better with the insulin/glucagon ratio $(r=0.965, p<0.001 ;$ Fig. $5 \mathrm{C})$. As to the lactate and pyruvate, serum glucose did not correlate with those of lactate $(r=-0.132$, n.s.; Fig. $5 D)$, but a very high significant relationship was found with pyruvate $(r=0.993, p<0.001$; Fig. 5E); indeed, glucose level also showed an inverse correlation with the lactate/pyruvate ratio $(r=-0.806, p<0.001 ; F i g .5 F)$, which indicates the relevance of fluctuations in pyruvate levels. In the same context, glucose did not significantly correlate with $\beta$-OH-but $(r=0.146$, n.s.; Fig. 5G), but correlated highly and directly with those levels for $A c A c(r=-0.963, p<0.001$; Fig. 5H). Moreover, glucose also showed an inverse correlation with the $\beta-\mathrm{OH}$-but /AcAc ratio $(r=-0.584, p<0.01$; Fig. 5l); therefore, glucose increase was highly correlated with an enhancement in oxidized metabolic products, namely pyruvate and AcAc (Fig. 5). 


\section{Effects of chronic exposure to EMF on rat blood glucose, lipids, and parameters indicative of redox state and energy status.}

Most of the reported effects of EMFs on cell proliferation, cell signaling, oxidant status, or metabolic changes have been obtained through chronic exposure to EMFs in animals or isolated cells [2]. Therefore, we searched whether the acute response to EMFs remains even after exposing the animals for 14 consecutive days to EMFs. For this, a set of rats was exposed for $15 \mathrm{~min}$ to $60 \mathrm{~Hz}$-EMFs per day for two weeks; then, rats were fasted overnight at the 15th day, and treated again for the same time. Table 1 shows that this chronically-treated group also elicited a significant hyperglycaemia, but the decrease in FFA serum levels was not seen. The lactate/pyruvate ratio was decreased and, consequently, the $\mathrm{NAD} / \mathrm{NADH}$ ratio was augmented in both groups, but through different mechanism; in the group of a single exposure, pyruvate levels were increased, whereas in the chronic group, the levels of lactate were diminished (Table 1). Total ketone bodies level was not significantly changed in both groups, but, in the chronic group, the $\beta-\mathrm{OH}$-but / AcAc ratio was decreased, indicating a more oxidized NAD/NADH status, presumably reflecting liver mitochondria redox state (Table 1). Finally, when examining blood energy parameters (ATP/ADP ratio, energy charge, and total adenine nucleotides), we did not find any significant difference in these indicators in either the "acute" or the "chronic" groups (Table 1). Therefore, chronic application of EMFs does not induce refractory actions on the effects of a single exposure of $60 \mathrm{~Hz}$-EMFs to rats. 
Table 1

Effects of chronic exposure to EMF on rat blood glucose, lipids, and parameters indicative of redox state and energy status.

\section{Fasted (single) Fasted (chronic)}

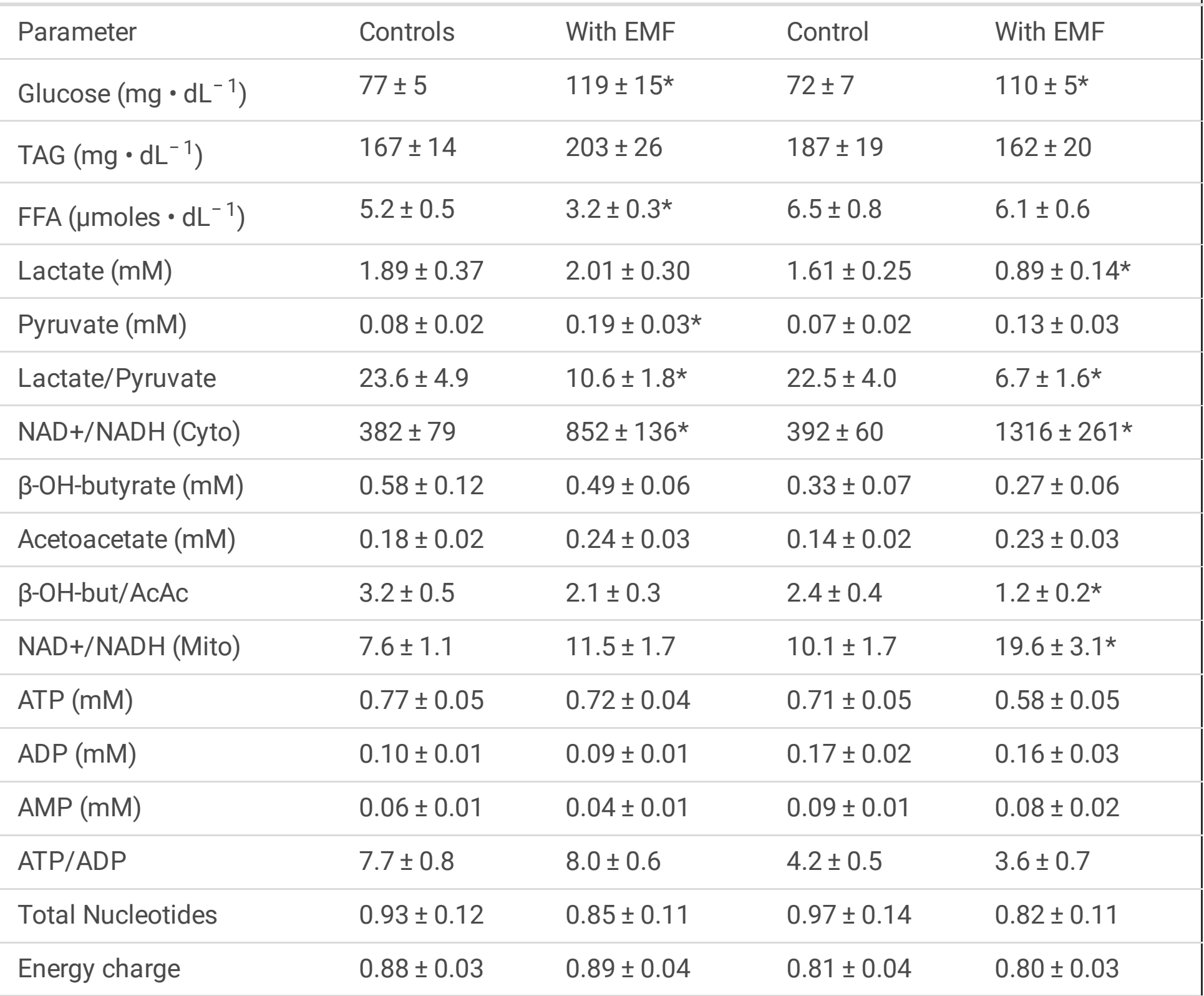

Results are the mean \pm SE of four individual observations per experimental group, which consisted in rats exposed to $60-\mathrm{Hz}$ EMFs during $15 \mathrm{~min} /$ day for 14 consecutive days before the single (acute) exposure at the day of the experiment, and blood samples were taken $30 \mathrm{~min}$ after ending exposure. Abbreviations: $\beta$-OH-but, $\beta$-hydroxybutyrate, AcAc, acetoacetate, Cyto, cytoplasm, and Mito, mitochondria. Statistical significance: ${ }^{*} p<0.01$ vs. control group.

\section{Discussion}

Interaction between biological systems and external EMFs has received considerable attention in the last times, but there are discrepant results that complicate reaching a conclusion on this interaction. We must take into account the complexity of biological systems as well as the different protocols in applying a variety of schemes for EMFs treatment [23]. In our experimental conditions, in normal fed or fasted male 
Wistar rats, we found that EMFs induced a hyperglycaemic state in both, fed and fasted rats, accompanied by a drastic decrease in serum FFA levels; these effects also coincided with an attenuation of a second serum insulin peak. Indeed, increased serum glucose levels were closely related with the cellular redox state, mainly favored by a more oxidized state and the insulin/glucagon ratio.

An increased, albeit slight, blood glucose level accompanied by a diminished insulin content has already been reported in rats chronically exposed to uniform constant EMFs [21]. This effect was related to an adrenergic effect induced by EMFs, where adrenaline would favor the stimulation of glucagon secretion over that of insulin at the level of pancreatic Langerhans islets [24, 25]. In this regard, there are clear discrepancies about the effects of EMFs on insulin secretion in rats, which seem to depend on the applied frequency and magnitude of the magnetic intensity flux [26, 27]. From here, that the in vitro experiments, where EMFs exposure increases cell number under apoptotic culture conditions of pancreatic cells, could lead to new therapeutic concepts in the treatment of diabetes [22].

The extremely low-frequency EMFs can affect $\mathrm{Ca} 2+$ mobilization and signaling, which can be involved in insulin release, and it has been found that $60-\mathrm{Hz}$ EMFs stimulation increases insulin secretion in diabetic and normal rats $[28,29]$. Indeed, it has been shown that EMFs with the same frequency of the natural frequencies of $\beta$-cell increase insulin secretion, which may indicate strong energy (ATP) coupling between insulin secretion and EMFs [23]. In contrast, EMFs can decrease glucose-stimulated insulin secretion and the ATP/ADP ratio in isolated cells, these effects are accompanied by a membrane depolarization and an increased cytosolic free calcium ion concentration [22]. In other conditions, increased levels of glucose were accompanied by significant variations in insulin secretion but without significant changes in glucagon levels after EMFs exposure (Fig. 2), which indicates that the insulin/glucagon ratio exerts more control in the glycaemia (Fig. 5), and appears as a main factor in the EMFs-mediated effect on blood glucose. The control of cholesterol by insulin and glucagon is regulated by plasma amino acids, being the insulin/glucagon ratio an early metabolic index controlling these effects [30]. In addition, an increased insulin/glucagon ratio after reperfusion correlates with the arterial ketone body ratio during recovery of graft liver function after in vivo liver transplantation [31].

Besides, chronic EMFs exposure is able to decrease the high levels of FFA and TG found in rats subjected to ischemia, probably by affecting FFA and TAG metabolism by enhancing availability of metabolic energy [18]. Moreover, the same research group reported previously that EMFs tend to suppress the stressinduced increase in plasma ACTH and glucose levels, reducing plasma lactate level, without affecting those of pyruvate [19]. In the present study, we found that EMFs decreased FFA levels without affecting those of TAG, even in a stressing state such as an overnight fast (Fig. 1); in addition, EMFs diminished lactate levels in fed animals whereas augmenting those of pyruvate (Fig. 2). On the contrary, in fasted rats, lactate was not decreased but pyruvate was significantly increased (Fig. 2); as a result, in both metabolic situations, the lactate/pyruvate ratio was diminished and, therefore, the NAD/NADH redox potential was significantly increased (Table 1). Besides, the phosphorylation potential and the mitochondrial functionally to carry out oxidative phosphorylation, the cell capacity to oxidize substrates would depend upon another thermodynamic factor, known as the oxidation-reduction (Redox) potential 
[32]. From here, cell redox state is recognized as an important regulatory factor of many metabolic fluxes [33].

We have shown that blood variations in the redox-pair metabolites, such as lactate and pyruvate, and mainly those in ketone bodies ( $\beta-\mathrm{OH}$-but and $\mathrm{AcAc})$, largely reflect those occurring in the liver [34]. Here, the effects of EMFs clearly favored a more oxidized cellular redox state, which could explain the in vivo changes in glucose metabolism in rats exposed to EMFs (Fig. 4). For instance, these animals showed an increased glucose oxidation, which we think was due to an insulin-dependent effect in the muscles, which was accompanied by decrements in glycogen synthesis (Fig. 4D), but was not seen in the liver coinciding with the abatement of the second insulin peak, and contrasting with the enhanced liver glycogen accumulation found after chronic EMFs exposure [35]. In the same context, we recorded a transient decrease in the rate of lipogenesis in the epididymal adipose tissue, which is also a metabolic pathway dependent on the insulin action (Fig. 4F); interestingly, EMFs promoted lipogenesis later in the liver (Fig. 4E), which was not seen due to effects of EMFs on TAG mobilization from the liver. Our data agree with previous results suggesting that putative effects on serum lipids are more significant in longer time periods of EMFs exposure, different frequencies, intensities, and durations of EMF exposure [36].

As mentioned above, another important factor involved in the metabolic effects induced by EMFs exposure can be the production of cell energetic parameters (ATP availability). Plasma glucose level respond to EMFs in the kilohertz range elevating insulin secretion, where EMFs would reach the internal structures and increase ATP synthesis [37, 38]. Indeed, exposure to EMFs attenuated KCl-stimulated insulin secretion influencing the increase in ATP/ADP and membrane depolarization, and attenuating the increase of $\left[\mathrm{Ca}^{2+}\right]$ i which is linked to a depolarization in isolated $\beta$-cells $[39,40]$. In our study, neither with a single (acute) nor with a chronic exposure to EMFs, did we detect any significant change in the energy parameters in the blood from these animals (Table 1), which would suggest that metabolic energy availability was not an important factor in the changes in glucose metabolism and insulin secretion elicited by EMFs under our experimental conditions. However, we have also demonstrated that changes in blood energetic parameters are very closely related with those in the liver [41]; therefore, we ignore whether our protocol for EMFs treatment actually affected pancreatic energy parameters in our experimental animals, similarly to what happens in isolated $\beta$-cells. This and other issues are indeed objectives for future research.

In conclusion, our data indicate that EMFs induced a hyperglycaemic state in both, fed and fasted rats, accompanied by a drastic attenuation of a second serum insulin peak. Indeed, increased serum glucose levels were closely related with the cellular redox state and with the insulin/glucagon ratio; moreover, glucose metabolism in the whole animal also coincided with variations in insulin and glucagon secretion, probably, dependent on the cell redox state. Most of these effects were also recorded in animals subjected to a chronic exposure to EMFs, indicating that there were no refractory actions for a continuous application of magneto-therapy.

\section{Material And Methods}




\section{Materials.}

Enzymes and coenzymes for enzyme-coupled reactions for metabolite determinations, as well as other analytical reagents, were purchased from Sigma-Aldrich Chemical Co. (St. Louis, MO, USA). The U $-{ }^{14} \mathrm{C}-$ glucose (sp. act. 9.9 GBq/mol; NEN Radiochemicals, Boston, MA, USA).

\section{Animal subjects and ethics.}

Our research was approved by the Animal Experiments Institutional Ethics Committee according to the Federal Regulations for Animal Experimentation (Ministry of Agriculture, SAGARPA, Mexico) and in compliment with the ARRIVE guidelines. Seventy male 10-week-old Wistar rats (240-270 g of b.w.) were obtained from the certified bioterium of the Instituto de Fisiología Celular, UNAM; animals were housed under a 12:12 h light/dark cycle with free access to food (diet chow 500I, LabDiet Co.) and water.

\section{Animal treatment (exposure to EMFs).}

The EF exposure system is composed of a high voltage transformer, a constant voltage unit, and EF exposure cages, which was designed for a rat or a smaller animal, as a cylindrical plastic cage. This cage has two stainless steel electrodes, connected to a plastic wrapped solenoid that laterally surrounded the cylindrical cage. We modulated the intensity of ELF-MF using the transformer. These coils produced a 60$\mathrm{Hz}$ magnetic field set to $3.8 \mathrm{mT}$ root mean square (rms) amplitude using a gaussmeter (Model 410; Lake Shore Cryotronics, Westerville, $\mathrm{OH}, \mathrm{USA}$ ) in the vertical direction at the centre of the system [42]. The experimental group was exposed to a single exposure at a magnetic intensity of $3.8 \mathrm{mT}, 60-\mathrm{Hz}$ extreme low frequency EMFs for $15 \mathrm{~min}$ in the cylindrical cage. Sham control animals were placed in the same location with the coils turned off, being only exposed to the local ambient geomagnetic field; the EMFs exposure was always done between 10:00 and 11:30 h. At indicated post-treatment (EMFs exposure) times, blood samples were collected through cardiac puncture and animals were euthanized with an overdose of sodium pentobarbital. Another set of rats was fasted overnight before treatment and euthanasia.

\section{Sera sampling and clinical tests.}

Serum was obtained by centrifuging uncoagulated whole blood; in these sera, glucose and triacylglycerols (TAG) were measured through standardized procedures with kits from SPINREACT (Spain). Serum levels of total fatty acids (FFA) were determined by $\mathrm{pH}$ titration, according to Novak [43]. Serum insulin and glucagon levels were measured with kits from RayBiotech (Peachtree Corners, GA, USA). 


\section{Curve of glucose tolerance.}

After an overnight fast, the animals were orally given $2 \mathrm{~g} \cdot \mathrm{kg}^{-1}$ of glucose and exposed $15 \mathrm{~min}$ to the generated EMFs. Blood samples were taken from a tail vein at 15, 30, 60, 120, and 180 min, after glucose administration ( $n=4$, per experimental point), placed in capillary tubes and centrifuged to obtain sera. Thereafter, in these serum samples, we performed the determinations of glucose, insulin, and glucagon.

\section{Analytical procedures.}

In neutralised with $4 \mathrm{~mol} \cdot \mathrm{L}^{-1} \mathrm{~K}_{2} \mathrm{CO}_{3}$ serum samples from perchloric acid-extracts, the redox-pair metabolites: lactate, pyruvate, $\beta$-hydroxybutyrate ( $\beta$-OH-but), and acetoacetate (AcAc) were enzymatically determined, as described before [34]. For adenine nucleotides (ATP, ADP, and AMP) determination, whole blood was directly poured for extraction into ice-cold $8 \%$ perchloric acid; after centrifugation, the sample was neutralized with $4 \mathrm{~mol} \cdot \mathrm{L}^{-1} \mathrm{~K}_{2} \mathrm{CO}_{3}$. Adenine nucleotides in the neutralized acid-extract were quantified by reversed-phase high performance liquid chromatography (HPLC) [44].

\section{In vivo quantification of $\left({ }^{14} \mathrm{C}\right)$-glucose oxidation and its incorporation to $\left({ }^{14} \mathrm{C}\right)$ - glycogen, as well as of $\left({ }^{14} \mathrm{C}\right)$-glycerol into lipid.}

A set of rats was intraperitoneally injected with $0.2 \mathrm{~mL}$ of saline-dissolved $0.5 \mathrm{mg}$ of glucose-containing 4 $\mu \mathrm{Ci}(148 \mathrm{mBq})$ of $\left(\mathrm{U}^{14} \mathrm{C}\right)$-glucose. After administration, animals were individually placed in the metabolic cage, especially designed for rats, and the experimental group was now exposed to $60 \mathrm{~Hz}$-EMFs for 15 min (time zero). Afterwards, animals were euthanized at 15, 60, and $120 \mathrm{~min}$, and samples were taken from serum, liver, caudo-femoralis muscle, and the epididymal white adipose tissue. The ${ }^{14} \mathrm{CO}_{2}$ was trapped using a strong base, hyamine hydrochloride $\left(100 \mathrm{mmoles} \cdot \mathrm{L}^{-1}\right)$, placed in an especial well in the metabolic cage. Glycogen (liver and muscle) was extracted and hydrolysed with $30 \% \mathrm{KOH}$ and saturated $\mathrm{Na}_{2} \mathrm{SO}_{4}$. The precipitated glycogen was re-suspended in distilled water, and incubated with $5 \%$ phenol and concentrated $\mathrm{H}_{2} \mathrm{SO}_{4}$ at room temperature, and the absorbance was measured at $490 \mathrm{~nm}$ [45]. The pattern of incorporated $\left({ }^{14} \mathrm{C}\right)$-glucose derived- $\left({ }^{14} \mathrm{C}\right)$-glycerol into total lipids from liver and epididymal adipose tissue was also evaluated. For this, total lipids were extracted from the tissue pellets with chloroform, and the neutral lipids and phospholipids were separated on silica gel thin-layer (TLC) plates. The TAG fraction was revealed by staining with iodine vapours and this lipid fraction was scraped into vials [46].

\section{Calculations and Statistics.}


Cytoplasmic and mitochondrial NAD/NADH ratios were calculated as follows: NAD/NADH = [oxidized substrate]/[reduced substrate] $\cdot 1 /$ Keq, taking into account equilibrium constants for lactate, and $\beta$ hydroxybutyrate dehydrogenases [47]. Linear regression analyses were made using the equation from Pearson's approach. Results are expressed as mean \pm SD, and statistical significance of the differences was assessed by two-way ANOVA for a normal distribution of data. Then, a Newman Keuls test was further applied and a $p<0.01$ value was considered as significant.

\section{Declarations}

\section{Data Availability.}

All data generated or analysed during this study are included in a Supplementary Information File).

\section{Acknowledgments:}

Authors thank to Ignacio Cabrera-Larios, MD, for the design and manufacturing of the electronic device for generating the EMFs. We also acknowledge the submission of the manuscript in Research Square in the following link: https://www.researchsquare.com/article/rs-83151/v1

\section{Disclosure Statement:}

The authors have no conflicts of interest to declare.

\section{Authors' contributions:}

All authors (G. M.-G., M. L.-C., and R. H.-M.) conceived and designed the experiments, performing the experiments. Similarly, all authors carried out the experiments, analysed the data and wrote the manuscript.

\section{References}

1. Moon, J. H. Health effects of electromagnetic fields on children. Clin. Exp. Pediatr.May 26, https://doi.org/10.3345/cep.2019.01494 (2020).

2. Karimi, A., Ghadiri Moghaddam, F. \& Valipour, M. Insights in the biology of extremely low-frequency magnetic fields exposure on human health. Mol. Biol. Rep.47, 5621-5633 (2020).

3. Gye, M. C. \& Park, C. J. Effect of electromagnetic field exposure on the reproductive system. Clin. Exp. Reprod. Med.39, 1-9 (2012).

4. Cichoń, N. et al. Benign effect of extremely low-frequency electromagnetic field on brain plasticity assessed by nitric oxide metabolism during poststroke rehabilitation. Oxid. Med. Cell. Longev.2017, 
2181942 (2017).

5. Mansourian, M., Firoozabadi, M. \& Hassan, Z. M. The role of 217-Hz ELF magnetic fields emitted from GSM mobile phones on electrochemotherapy mechanisms. Electromagn. Biol. Med.39, 239249 (2020).

6. Zwolińska, J., Gąsior, M., Śnieżek, E. \& Kwolek, A. The use of magnetic fields in treatment of patients with rheumatoid arthritis. Review of the literature. Reumatologia.54, 201-206 (2016).

7. Cichoń, N. et al. Modulation of antioxidant enzyme gene expression by extremely low frequency electromagnetic field in post-stroke patients. Scand. J. Clin. Lab. Invest.78, 626-631 (2018).

8. Yuan, J., Xin, F. \& Jiang, W. Underlying signaling pathways and therapeutic applications of pulsed electromagnetic fields in bone repair. Cell. Physiol. Biochem.46, 1581-1594 (2018).

9. Vincenzi, F. et al. Pulsed Electromagnetic Field Exposure Reduces Hypoxia and Inflammation Damage in Neuron-Like and Microglial Cells. J. Cell. Physiol.232, 1200-1208 (2017).

10. Li, R. L. et al. Pulsed electromagnetic field improves postnatal neovascularization in response to hindlimb ischemia. Am. J. Transl. Res.7, 430-444 (2015).

11. Pena-Philippides, J. C. et al. Effect of pulsed electromagnetic field (PEMF) on infarct size and inflammation after cerebral ischemia in mice. Transl. Stroke Res.5, 491-500 (2014).

12. Urnukhsaikhan, E., Mishig-Ochir, T., Kim, S. C., Park, J. K. \& Seo, Y. K. Neuroprotective effect of low frequency-pulsed electromagnetic fields in ischemic stroke. Appl. Biochem. Biotechnol.181, 13601371 (2017).

13. Gessi, S. et al. Pulsed electromagnetic field and relief of hypoxia-induced neuronal cell death: The signaling pathway. J. Cell. Physiol.17, https://doi.org/10.1002/jcp.28149 (2019 Jan).

14. Sun, W., Gan, Y., Fu, Y., Lu, D. \& Chiang, H. An incoherent magnetic field inhibited EGF receptor clustering and phosphorylation induced by a $50-\mathrm{Hz}$ magnetic field in cultured FL cells. Cell. Physiol. Biochem.22, 507-514 (2008).

15. Park, J. E. et al. Electromagnetic fields induce neural differentiation of human bone marrow derived mesenchymal stem cells via ROS mediated EGFR activation. Neurochem. Int.62, 418-424 (2013).

16. Mattsson, M. O. \& Simkó, M. Grouping of Experimental Conditions as an approach to evaluate effects of extremely low-frequency magnetic fields on oxidative response in in vitro studies. Front. Public Health.2;2, 132 https://doi.org/10.3389/fpubh.2014.00132 (2014 Sep).

17. Cichoń, N., Bijak, M., Miller, E. \& Saluk, J. Extremely low frequency electromagnetic field (ELF-EMF) reduces oxidative stress and improves functional and psychological status in ischemic stroke patients. Bioelectromagnetics.38, 386-396 (2017).

18. Harakawa, S. et al. Effects of exposure to a $50 \mathrm{~Hz}$ electric field on plasma levels of lactate, glucose, free fatty acids, triglycerides and creatine phosphokinase activity in hind-limb ischemic rats. J. Vet. Med. Sci.67, 969-974 (2005).

19. Harakawa, S., Takahashi, I., Doge, F. \& Martin, D. E. Effect of a $50 \mathrm{~Hz}$ electric field on plasma ACTH, glucose, lactate, and pyruvate levels in stressed rats. Bioelectromagnetics.25, 346-351 (2004). 
20. Hori, T. et al. Effect of $50 \mathrm{~Hz}$ electric field in diacylglycerol acyltransferase mRNA expression level and plasma concentration of triacylglycerol, free fatty acid, phospholipid and total cholesterol.Lipids Health Dis. 2012 Jun7;11:68. doi: 10.1186/1476-511X-11-68.

21. Gorczynska, E. \& Wegrzynowicz, R. Glucose homeostasis in rats exposed to magnetic fields. Invest. Radiol.26, 1095-1100 (1991).

22. Sakurai, T., Yoshimoto, M., Koyama, S. \& Miyakoshi, J. Exposure to extremely low frequency magnetic fields affects insulin-secreting cells. Bioelectromagnetics.29, 118-124 (2008).

23. Farashi, S. Interaction between pancreatic $\beta$ cell and electromagnetic fields: A systematic study toward finding the natural frequency spectrum of $\beta$ cell system. Electromagn. Biol. Med.36, 341-356 (2017).

24. Barnothy, M. F. \& Sumegi, I. Abnormalities in organs of mice induced by a magnetic field. Nature.221 (5177), 270-271 (1969).

25. Dixey, R. \& Rein, G. 3H-noradrenaline release potentiated in a clonal nerve cell line by low-intensity pulsed magnetic fields. Nature.296 (5854), 253-256 (1982).

26. Sutter, B. C., Billaudel, B., Sutter-Dub, M. T. \& Bellossi, A. Effects of constant magnetic fields on the Bcells and insulin target cells in the rat. Aviat. Space Environ. Med.58, 537-540 (1987).

27. Kost, J., Wolfrum, J. \& Langer, R. Magnetically enhanced insulin release in diabetic rats. J. Biomed. Mater. Res.21, 1367-1373 (1987).

28. Adeghate, E., Ponery, A. S. \& Wahab, A. Effect of electrical field stimulation on insulin and glucagon secretion from the pancreas of normal and diabetic rats. Horm. Metab. Res.33, 281-289 (2001).

29. Cha, C. Y. et al. Ionic mechanisms and $\mathrm{Ca} 2+$ dynamics underlying the glucose response of pancreatic $\beta$ cells: a simulation study. J. Gen. Physiol.138, 21-37 (2011).

30. Sanchez, A. \& Hubbard, R. W. Plasma amino acids and the insulin/glucagon ratio as an explanation for the dietary protein modulation of atherosclerosis. Med. Hypotheses.35, 324-329 (1991).

31. Ichimiya, M. et al. Insulin and glucagon levels in living related liver transplantation: their interaction with the recovery of graft liver function. Transpl. Int.8, 165-168 (1995).

32. Azzone, G. F., Zoratti, M., Petronilli, V. \& Pietrobon, D. The stoichiometry of $\mathrm{H}+$ pumping in cytochrome oxidase and the mechanism of uncoupling. J. Inorg. Biochem.23, 349-356 (1985).

33. Nicholl, D. G. Quantitative bioenergetics: The measurement of driving forces. In: Nicholl DG, Ferguson editors. Bioenergetics 2, Academic Press, Harcourt Brace Jovanich, Publishers 1992, p. 305 - 12.

34. Hernández-Muñoz, R. \& Díaz-Muñoz, M. \& Chagoya de Sánchez, V. Possible role of cell redox state on collagen metabolism in carbon tetrachloride-induced cirrhosis as evidenced by adenosine administration to rats. Biochim. Biophys. Acta.1200, 93-99 (1994).

35. Gorczynska, E. \& Wegrzynowicz, R. Structural and functional changes in organelles of liver cells in rats exposed to magnetic fields. Environ. Res.55, 188-198 (1991).

36. Wang, Z. et al. Effects of electromagnetic fields on serum lipids in workers of a power plant. Environ. Sci. Pollut. Res. Int.23, 2495-2504 (2016). 
37. Tsong, T. Y. \& Astumian, R. D. Electroconformational coupling: how membrane-bound ATPase transduces energy from dynamic electric fields. Annu. Rev. Physiol.50, 273-290 (1988).

38. Havas, M. Dirty electricity elevates blood sugar among electrically sensitive diabetics and may explain brittle diabetes. Electromagn. Biol. Med.27, 135-146 (2008).

39. Sakurai, T., Koyama, S., Komatsubara, Y., Jin, W. \& Miyakoshi, J. Decrease in glucose-stimulated insulin secretion following exposure to magnetic fields. Biochem. Biophys. Res. Commun.332, 28-32 (2005).

40. Leibiger, B., Moede, T., Uhles, S., Berggren, P. O. \& Leibiger, I. B. Short-term regulation of insulin gene transcription. Biochem. Soc. Trans.30, 312-317 (2002).

41. Hernández-Muñoz, R. \& Chagoya de Sánchez, V. In vivo correlation between liver and blood energy status as evidenced by chronic treatment of carbon tetrachloride and adenosine to rats. Can. J. Physiol. Pharmacol.72, 1252-1256 (1994).

42. Cho, S. I. et al. Extremely low-frequency magnetic fields modulate nitric oxide signaling in rat brain. Bioelectromagnetics.33, 568-574 (2012).

43. Novak, M. Colorimetric ultramicro method for the determination of free fatty acids. J. Lipid Res.6, 431-433 (1965).

44. Hoffman, N. E. \& Liao, J. C. Reversed phase high performance liquid chromatographic separations of nucleotides in the presence of solvophobic ions. Anal. Chem.49, 2231-2234 (1977).

45. Hara, Y. \& Watanabe, N. Changes in expression of genes related to glucose metabolism in liver and skeletal muscle of rats exposed to acute hypoxia. Heliyon.6 (7), e04334 (2020).

46. Olguín-Martínez, M., Hernández-Espinosa, D. R. \& Hernández-Muñoz, R. High a-tocopherol dosing increases lipid metabolism by changing redox state in damaged rat gastric mucosa and liver after ethanol treatment. Clin. Sci. (London).132, 1257-1272 (2018).

47. Hernández-Muñoz, R. \& Contreras-Zentella, M. L. Involvement of cell oxidant status and redox state in the increased non-enzymatic ethanol oxidation by the regenerating rat liver. Biochem. Pharmacol.161, 63-72 (2019).

\section{Figures}




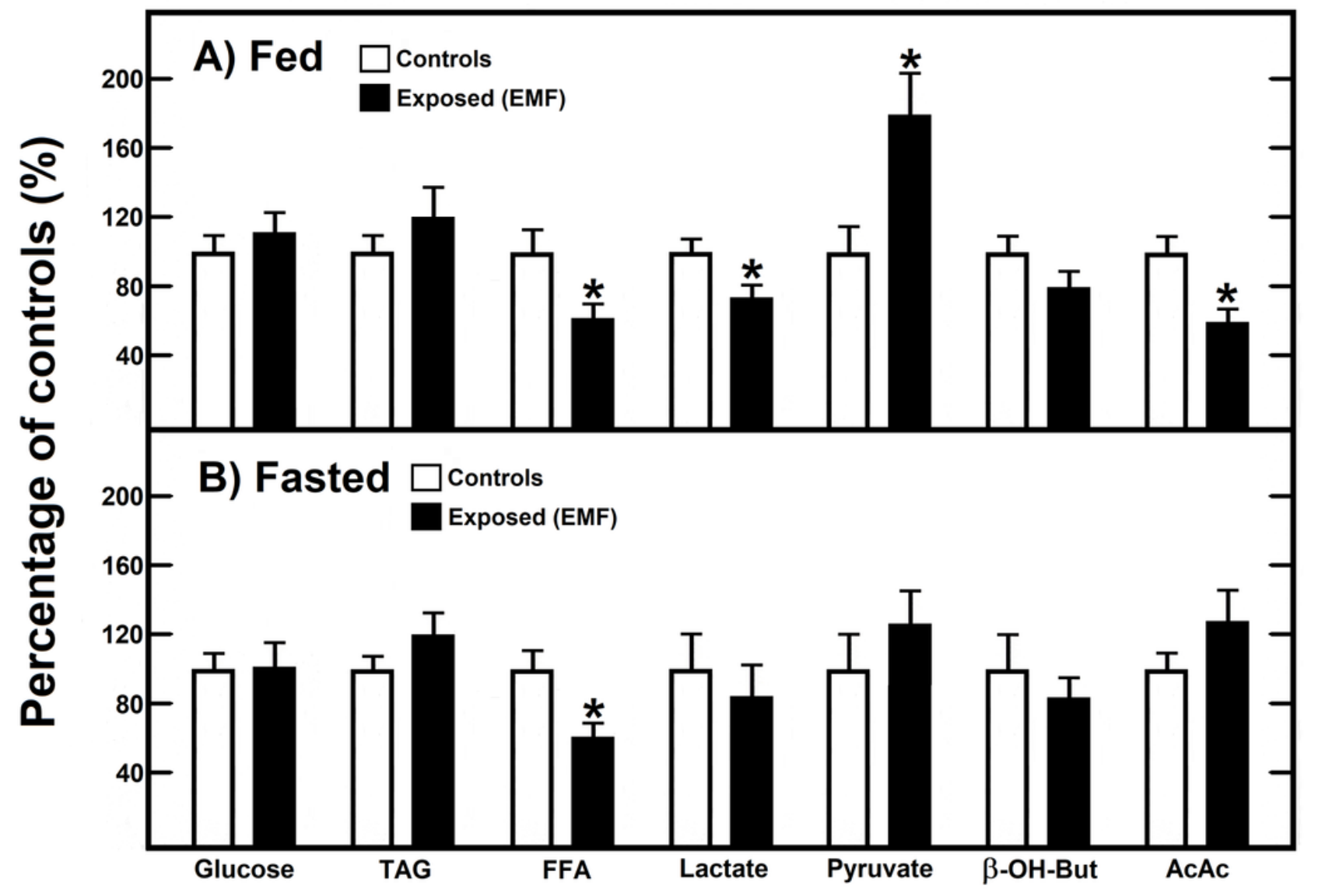

Figure 1

Effects of the single exposure to EMFs on glucose, lipids, and redox pair-metabolites in fed and fasted rats. Results are the mean \pm SE of four individual observations per experimental group. Blood samples were immediately taken after 15 min exposure to EMFs. Experimental groups are designated by symbols at the upper part of each panel. Control values (100\%) were, for fed animals, glucose $=101 \pm 9 \mathrm{mg} \cdot \mathrm{dL}-1$, $\mathrm{TAG}=174 \pm 15 \mathrm{mg} \cdot \mathrm{dL}-1, \mathrm{FFA}=3.8 \pm 0.5 \mu$ moles $\cdot \mathrm{dL}-1$, lactate $=1.26 \pm 0.08 \mathrm{mmoles} \cdot \mathrm{L}-1$, pyruvate $=$ $0.06 \pm 0.01$ mmoles $\cdot L-1$, $\beta$-hydroxybutyrate $(\beta-\mathrm{OH}$-but $)=0.16 \pm 0.02$ mmoles $\cdot L-1$, and acetoacetate $(A c A c)=0.32 \pm 0.06$ mmoles $\cdot L-1$. In fasted animals, values were: glucose $=77 \pm 5 \mathrm{mg} \cdot \mathrm{dL}-1, \mathrm{TAG}=167$ $\pm 14 \mathrm{mg} \cdot \mathrm{dL}-1, \mathrm{FFA}=5.2 \pm 0.5$ moles $\cdot \mathrm{dL}-1$, lactate $=1.89 \pm 0.37$ mmoles $\cdot \mathrm{L}-1$, pyruvate $=0.08 \pm 0.02$ mmoles $\cdot L-1, \beta$-hydroxybutyrate $(\beta-O H$-but $)=0.58 \pm 0.12$ mmoles $\cdot L-1$, and acetoacetate $(\mathrm{AcAc})=0.18 \pm$ 0.02 mmoles $\cdot L-1$. Statistical significance: ${ }^{*} p<0.01$ vs. control group. 


\section{FIGURE 2}

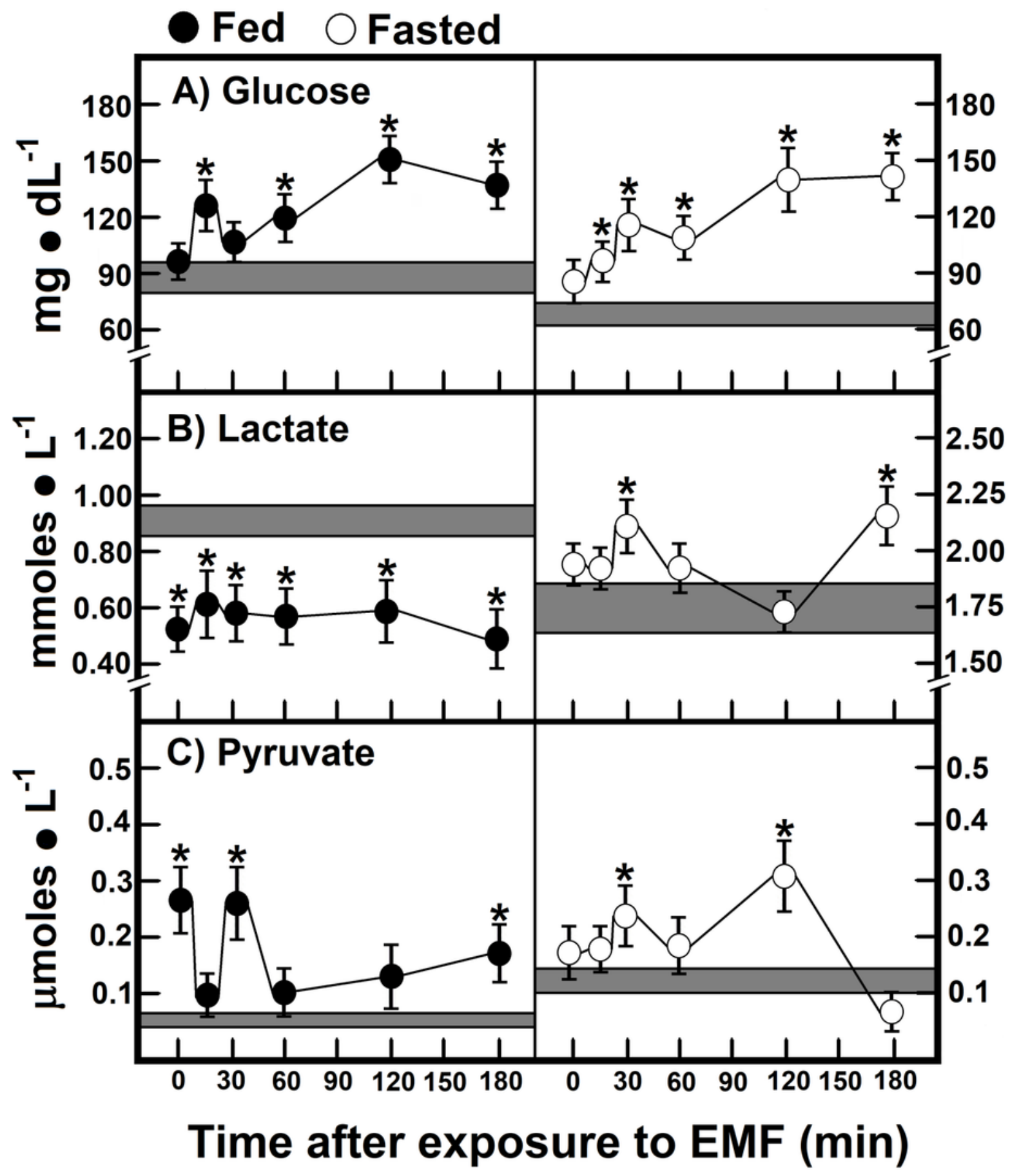

Figure 2

Time-course of EMFs effects on glucose, lactate, and pyruvate in fed and fasted rats. Results are the mean $\pm S E$ of four individual observations per experimental group and point, for glucose (panels $A$ ), lactate (panels B), and pyruvate (panels C). Experimental groups are designated by symbols at the upper part of the Figure. Statistical significance: ${ }^{*} p<0.01$ vs. control group. 


\section{FIGURE 3}

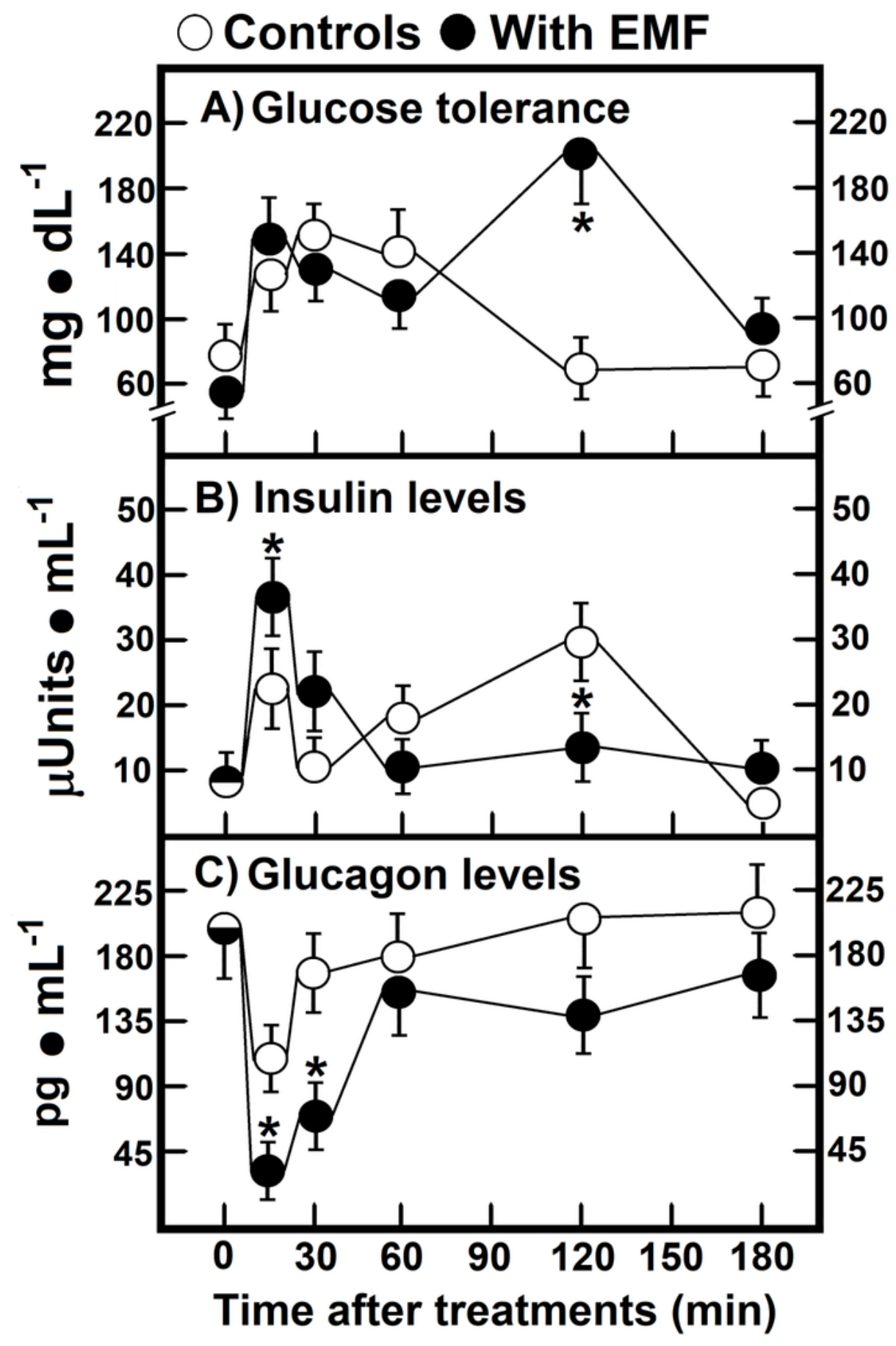

Figure 3

Curve of tolerance to glucose and levels of insulin and glucagon in rats treated with EMFs. Results are the mean \pm SE of four individual observations per experimental group and point, for glucose (panel $A$ ), insulin (panel B), and glucagon (panel C). Experimental groups are designated by symbols at the upper part of the Figure. Statistical significance as indicated in Fig. 1. 


\section{FIGURE 4}

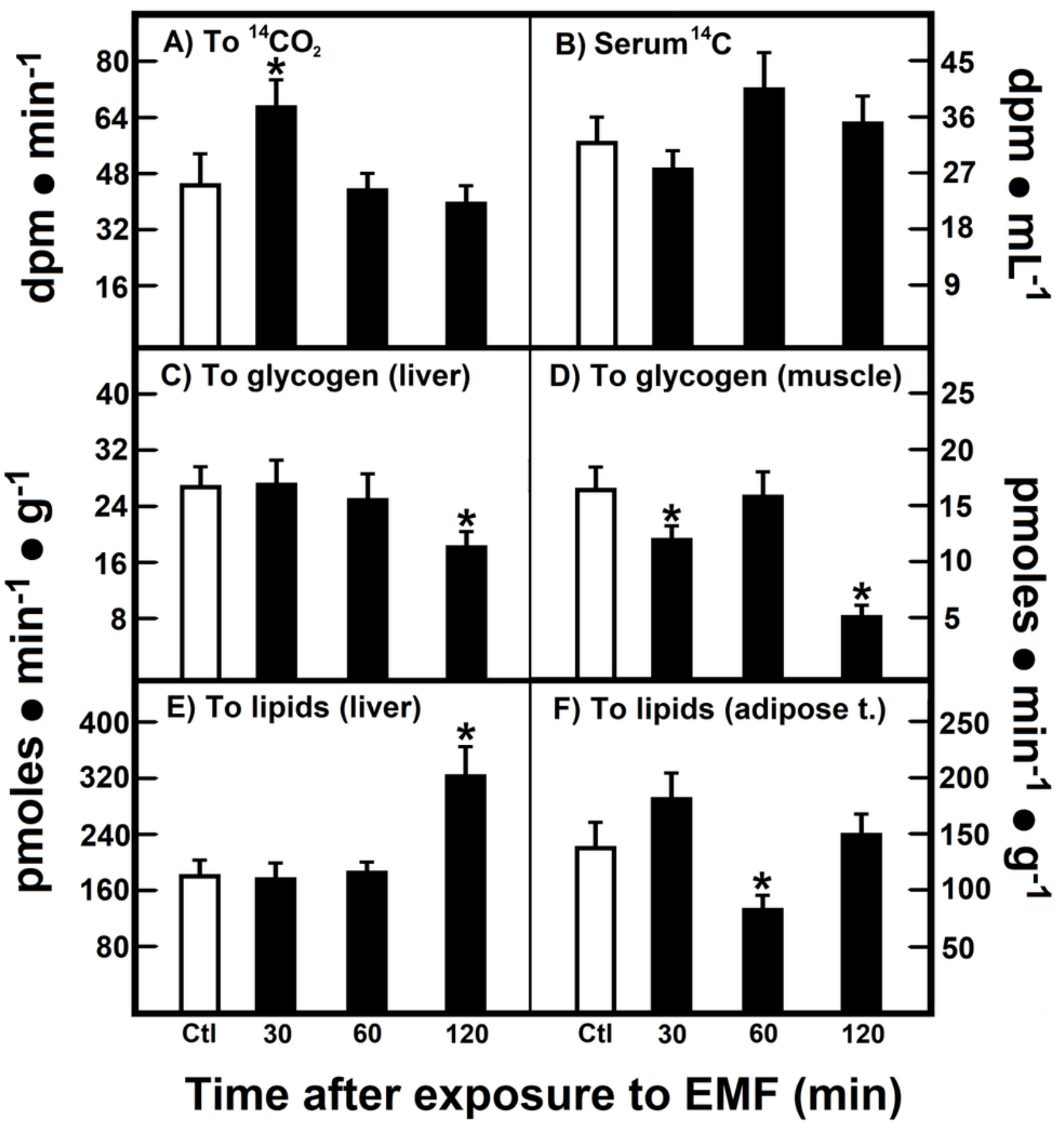

Figure 4

In vivo oxidation of U-(14C)-glucose and its incorporation into glycogen and glycerol-containing lipids in rats treated with EMFs. Results are the mean \pm SE of four individual observations per experimental group and point, for glucose oxidation (panel A), blood glucose (panel B), its incorporation into glycogen in the liver (panel C) and in the muscle (panel D). In addition, incorporation of labelled glycerol into TG is also 


\section{FIGURE 5}

\section{Controls With EMF}
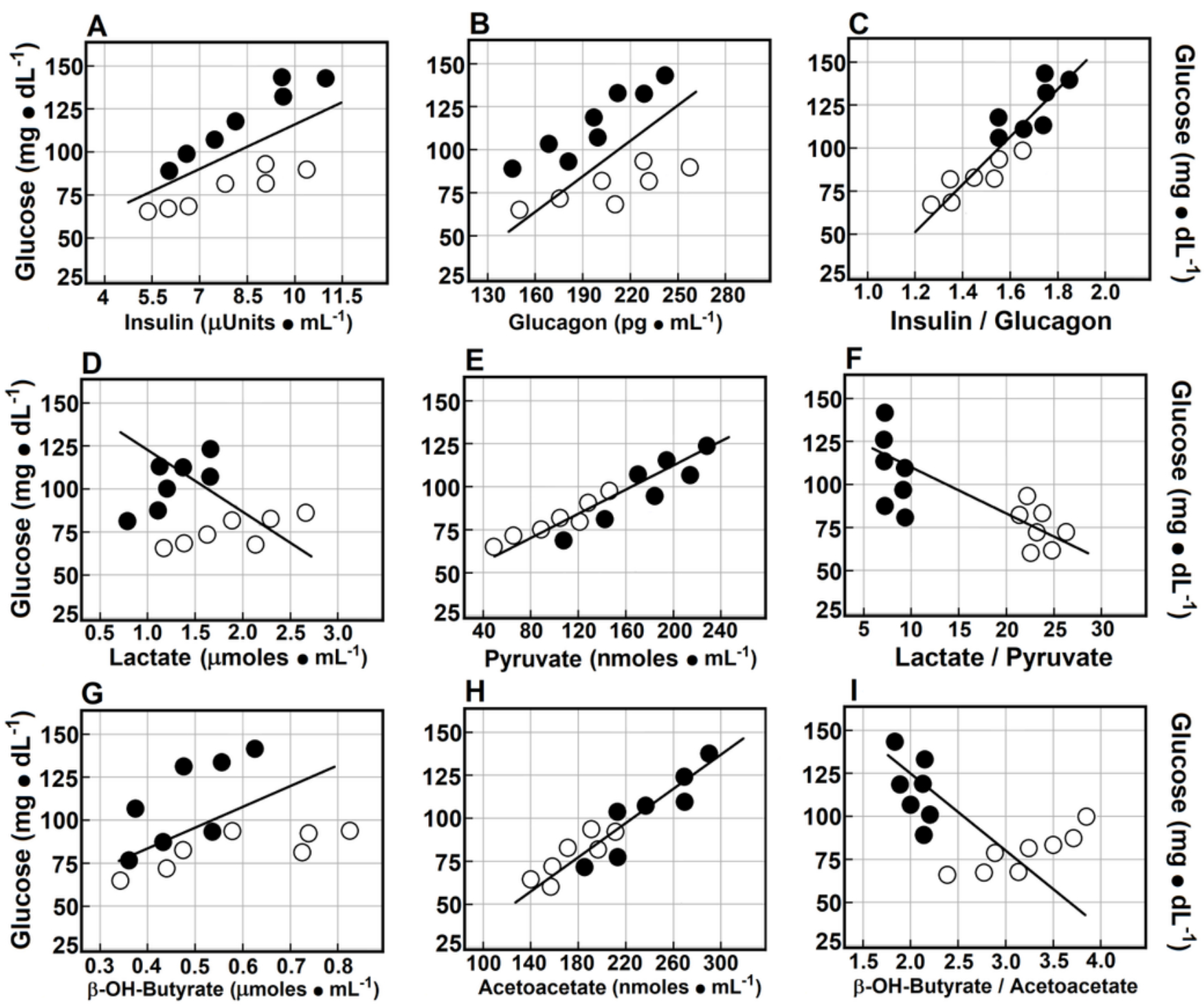

Figure 5

Correlations among serum glucose levels, insulin, glucagon, and redox- pair metabolites. Scatter plots show the relation between two sets of data. This relation is summarized in the Pearson's correlation coefficient ( $r$ ) for each relation in each scatter plot: panel A, glucose vs. insulin levels $(r=0.687, p<0.01)$, 
panel B: glucose vs. glucagon levels $(r=0.403, p<0.05)$, and panel $C$, glucose against the insulin/glucagon ratio $(r=0.965, p<0.001)$. Panel $D$ shows correlations of glucose vs. lactate levels $(r=$ -0.132 , n.s.), panel $E$, glucose against pyruvate levels $(r=0.993, p<0.001)$, and panel $F$, glucose levels vs. the lactate/pyruvate ratio $(r=-0.806, p<0.001)$. Finally, glucose against $\beta$-OH-but levels $(r=0.146, n . s)$ in panel $G$, panel $H$, glucose vs. AcAc levels $(r=-0.963, p<0.001)$, and panel $I$, glucose levels against the $\beta$ OH-but/AcAc ratio $(r=-0.584, p<0.01)$.

\section{Supplementary Files}

This is a list of supplementary files associated with this preprint. Click to download.

- SupplementalDataAvailability.txt

- SupplementalDataAvailability.pdf 